\title{
Arztgeheimnis: Kann sich der Arzt, der sich an einen Anwalt wendet, auf Notstand berufen?
}

Martin Schubarth

Altbundesrichter, Avocat-Conseil

Korrespondenz:

Prof. Dr. iur. Martin Schubarth

Rusconi \& Associés

4, rue de la Paix

CH-1002 Lausanne

www.martinschubarth.ch

\section{Ausgangspunkt: ein praktischer Fall}

Ein Patient kündigt seinem Arzt rechtliche Schritte wegen einer behaupteten Fehlbehandlung an. Der Arzt sucht einen Anwalt auf, um die Rechtslage zu erörtern und die gegebenenfalls notwendigen Schritte zu seiner Verteidigung in einem Zivil- oder Strafverfahren vorzubereiten. Darf der Arzt dem Anwalt Fakten mitteilen, die unter das Arztgeheimnis fallen; darf er ihm also gegebenenfalls Einblick in die Krankengeschichte geben?

Ein Kantonsarzt, dem diese Frage vom konsultierten Anwalt vorgelegt wurde, antwortete darauf wie folgt: Wenn der Patient den Arzt nicht vom Arztgeheimnis entbinde, müsse der Arzt sich an die Aufsichtsbehörde wenden. Diese könne ihn vom Arztgeheimnis entbinden, da das Interesse des Arztes (gemeint an der Aufhebung des Arztgeheimnisses) ebenso wichtig sei wie das des Patienten (an der Wahrung des Arztgeheimnisses auch gegenüber dem Anwalt des Arztes).

Die Antwort übergeht, ob der Arzt wirklich gezwungen ist, sich zunächst an die Aufsichtsbehörde zu wenden, oder ob er sich für die Offenbarung von Fakten, die unter das Arztgeheimnis fallen, direkt auf Notstand berufen kann, soweit die Weitergabe dieser Informationen an den Anwalt zur Abklärung der Rechtslage und der Vorbereitung der Verteidigung notwendig ist. Dieser Frage sei hier nachgegangen.

\section{Rechtslage zum rechtfertigenden Notstand}

Nach Art. 17 StGB betreffend den rechtfertigenden Notstand ist ein an sich strafbares Verhalten rechtmässig, wenn der Täter handelt, um ein Rechtsgut aus einer unmittelbaren, nicht anders abwendbaren Gefahr zu retten, soweit er dabei höherwertige Interessen wahrt.

In unserem Zusammenhang stellt sich die Frage, ob der Rückgriff auf den allgemeinen Rechtfertigungsgrund des Notstandes durch die Sonderregelung von Art. 321 Ziff. 2 StGB eingeschränkt ist. Danach ist die Verletzung des Arztgeheimnisses nicht strafbar, wenn die Aufsichtsbehörde den Arzt auf sein Gesuch hin schriftlich vom Arztgeheimnis entbunden hat.
Die Frage des Verhältnisses von Art. 17 StGB und Art. 321 Ziff. 2 StGB, also ob Art. 321 Ziff. 2 StGB den Anwendungsbereich von Art. 17 StGB, das heisst das Notstandsrecht, einschränkt, wird unterschiedlich beantwortet. Teils wird angenommen, die direkte Berufung auf Notstand sei zulässig [1]. Teils wird vertreten, dies sei zweifelhaft, wenn die Anrufung der Aufsichtsbehörde möglich ist [2] oder wenn diese das Gesuch ablehnt [3]. Teils findet sich die Auffassung, die Berufung auf Notstand komme nur subsidiär in dringenden Fällen in Betracht und diesfalls müsse möglichst rasch der Patient oder die Aufsichtsbehörde nachträglich um Entbindung vom Geheimnis angegangen werden [4, 5].

Grundsätzlich hat jedermann das Recht, sich in Fragen seiner beruflichen Tätigkeit rechtlich beraten zu lassen, sich insbesondere an einen Anwalt seiner Wahl zu wenden. Dies gilt auch für den Arzt und muss insbesondere gelten in einer Zeit, in der Fragen der rechtlichen Verantwortlichkeit des Arztes, seiner zivilen und seiner strafrechtlichen Haftung [6] vermehrt aktuell werden. Es gibt keinen sachlichen Grund, den Arzt in dieser Hinsicht schlechter zu stellen als andere Bürger und zu verlangen, dass er zunächst den Patienten oder die Aufsichtsbehörde um Entbindung vom Geheimnis ersuchen muss.

Allerdings hat der Arzt bei der Wahrnehmung seines Rechtes, einen Anwalt beizuziehen, auf andere Interessen, etwa Persönlichkeitsrechte anderer und Geheimhaltungspflichten Rücksicht zu nehmen. Soweit es nur um die Einholung genereller rechtlicher Auskunft geht, wird deshalb eine detaillierte Information unter Nennung des Patienten und Überlassung des medizinischen Dossiers nicht notwendig sein. Gegebenenfalls müsste das Dossier anonymisiert werden.

Anders verhält es sich in der Regel, wenn der Patient einen Arzt rechtlich zu belangen droht oder der Arzt mit dieser Möglichkeit rechnen muss. Hier sind Konstellationen denkbar, bei denen der Anwalt den Arzt nur aufgrund einer umfassenden Information über alle Einzelheiten des Falles beraten kann. Dies gilt insbesondere, wenn in einer Stellungnahme auf konkrete Einzelheiten der Krankengeschichte Bezug genommen werden muss. Hier stellt sich die Frage, ob es 
zulässig ist, dem Anwalt alle medizinischen Unterlagen, insbesondere die Krankengeschichte, auszuhändigen.

Entsprechende Fragen können sich auch bei anderen Geheimnisträgern stellen, und zwar nicht nur bei solchen, die ebenfalls in StGB 321 genannt sind, sondern etwa auch bei Trägern von Amts-, Geschäfts- oder Bankgeheimnissen. Soweit hier ein Gesuch um Entbindung nicht möglich ist, weil keine Aufsichtsbehörde oder kein Art. 321, Ziff. 2 StGB entsprechendes Verfahren besteht, ist der direkte Rückgriff auf Grundsätze des rechtfertigenden Notstandes unausweichlich [7]. Dies spricht dafür, dass sich auch ein Arzt direkt auf diesen Rechtfertigungsgrund berufen kann.

\section{Weitere Folgen eines Gesuches an die Aufsichtsbehörde}

Es kommt folgender Gesichtspunkt hinzu: Wenn der Arzt zunächst ein Gesuch an die Aufsichtsbehörde einzureichen hat, ist er auch in diesem Verfahren gezwungen, gegebenenfalls auf Gesichtspunkte hinzuweisen, die unter das Arztgeheimnis fallen. Das bedeutet aber, dass das Arztgeheimnis gegenüber einem grösseren Personenkreis gelüftet wird [8], als wenn sich der Arzt direkt, ohne den Umweg über ein Gesuchsverfahren, an seinen Anwalt wendet. Ob damit dem Patienten gedient ist, ist zu bezweifeln.

Die Notwendigkeit eines Gesuches an die Aufsichtsbehörde ist überdies problematisch, wenn der Arzt sich im Gesuch gegebenenfalls selbst belasten müsste. Besonders pikant ist dieser Gesichtspunkt, wenn, wie etwa im Kanton VD, der erste Staatsanwalt Mitglied der Aufsichtsbehörde ist [9].

$\mathrm{Zu}$ beachten ist auch das Zeitmoment: Die Behandlung des Gesuches durch die Aufsichtsbehörde kann die für den Arzt unter Umständen dringliche Kontaktnahme mit dem Anwalt verzögern. Wenn man aber für Fälle der Dringlichkeit ausnahmsweise einen direkten Rückgriff auf Notstand zulässt, dann wird deutlich, dass letztlich das materielle Notstandsrecht massgeblich ist für die Rechtfertigung der Weitergabe von Informationen, die unter das Arztgeheimnis fallen. Dies zeigt, dass die Möglichkeit des Verfahrens nach Art. 321, Ziff. 2 StGB den direkten Rückgriff auf Notstand nicht ausschliesst.

Es kommen weitere Gesichtspunkte hinzu, die das Gesuchsverfahren betreffen. Wer einen Anwalt aufsucht, um sich über seine rechtliche Situation zu orientieren, tut dies in der Regel ohne Wissen der (potentiellen) Gegenpartei. Die Möglichkeit, sich an eine Person seines Vertrauens zu wenden, ohne andere vorgängig dar- über informieren zu müssen, ist dem Persönlichkeitsrecht inhärent. Insbesondere die (potentielle) Gegenpartei braucht nicht zu wissen, ob und wann sich der Arzt an einen Anwalt wenden will.

Nun wird aber angenommen, dass in einem Gesuchsverfahren die Aufsichtsbehörde den Patienten in der Regel anzuhören hat, und dies ist offenbar in einem Teil der Kantone auch gängige Praxis [10]. Kann sich der Arzt dagegen ohne Einschränkung auf das Notstandsrecht berufen, entfällt dieser praktische Zwang, seinen potentiellen Gegner über seine Schritte zu informieren.

Die Sache wird noch brisanter, wenn man berücksichtigt, dass der Entscheid der Aufsichtskommission wie jeder Verwaltungsentscheid einem Rechtsmittelverfahren unterliegt [11], der Patient also einen positiven Gesuchsentscheid gerichtlich anfechten kann.

\section{Kriterien der Rechtfertigung des Notstandes}

Zu prüfen sind deshalb die Kriterien der Rechtfertigung des Notstandes in der hier erörterten Konstellation.

Notstand ist gekennzeichnet durch eine Güterkollision. Vorliegend geht es einerseits um das Interesse des Patienten an einer strikten Wahrung des Geheimnisses, das heisst an allem, was er dem Arzt an persönlichen Informationen anvertraut hat oder was dieser in Ausübung seiner Tätigkeit wahrgenommen hat [12]. Andererseits geht es darum, dass der Arzt in einem Konflikt mit seinem Patienten nicht wehrlos dastehen muss, sondern die gleichen Möglichkeiten haben sollte, sich zu wehren, wie jeder andere von einem Prozess bedrohte Bürger. Der Arzt ist durch die Möglichkeit, dass gegen ihn rechtliche Schritte eingeleitet werden könnten, in seinen finanziellen Interessen, gegebenenfalls bei einem drohenden Strafverfahren in seinen Persönlichkeitsrechten und möglicherweise in seiner Berufsfreiheit bedroht. Dass er in einer solchen Situation im Rahmen des Notwendigen einen Anwalt seines Vertrauens über Tatsachen in Kenntnis setzt, die unter das Anwaltsgeheimnis fallen, ist deshalb gerechtfertigt [13]. Dabei ist zu beachten, dass der Anwalt seinerseits von den erhaltenen Informationen nur in dem Ausmass Gebrauch machen darf, die zur Wahrung der Interessen seines Klienten notwendig sind. Ein weiterer Gesichtspunkt könnte je nach Fallkonstellation sein, dass die Beeinträchtigung der Rechtsgüter des Arztes auf das Vorgehen des Patienten zurückzuführen ist, dieser also mit der Kontaktnahme des Arztes mit einem Anwalt rechnen muss. 
$\mathrm{Zu}$ unterstreichen ist allerdings: Stets ist die Frage der Anonymisierung von Patienteninformationen zu prüfen. Soweit eine sachgerechte anwaltliche Beratung gestützt auf anonymisierte Unterlagen möglich ist und die Anonymisierung sich ohne unverhältnismässigen Aufwand durchführen lässt, muss anonymisiert werden. Anders verhält es sich, wenn der Patient etwa durch Einleitung gerichtlicher Schritte seine Anonymität selbst gelüftet hat.

Als Fazit ist also festzuhalten, dass das Handeln des Arztes, der sich an einen Anwalt wendet und diesem im Rahmen des Notwendigen Tatsachen offenbart, die unter das Arztgeheimnis fallen, durch Notstand gerechtfertigt ist. Das Notstandsrecht wird durch die Möglichkeit eines Gesuchverfahrens nach Art. 321 Ziff. 2 StGB nicht eingeschränkt.

\section{Literatur}

1 So Bernard Corboz, Le secret professionnel de l'avocat selon art. 321 CP, SJ 1993, 77 ff., $97 \mathrm{mit}$ Hinweisen; Jörg Rehberg, Arzt und Strafrecht, in: Heinrich Honsell (Hrsg.), Handbuch des Arztrechtes, Zürich 1994, 303 ff., 348; Günter Stratenwerth, Strafrecht BT II 5. A. Bern 2000, 385; wohl ebenso Brigitte Tag, Die Verschwiegenheit des Arztes im Spiegel des Strafgesetzbuches der Strafprozessordnung des Kantons Zürich, ZStrR 2004, 1 ff., 13; dieselbe, Strafrecht im Arztalltag, in: Moritz Kuhn/Tomas Poledna (Hrsg.), Arztrecht in der Praxis, Zürich 2007, 669 ff., 753.

2 So Bernard Corboz, Les infractions en droit suisse II, Berne 2002, 659 f.; Andreas Donatsch/Wolfgang Wohlers, Strafrecht IV, 3. A. Zürich 2004, 488; Karin Keller, Das ärztliche Berufsgeheimnis gemäss Art. 321 StGB unter besonderer Berücksichtigung des Kantons Zürich, Zürich 1993, 167.

3 So Bernard Corboz, Les infractions en droit suisse II, Berne 2002, $659 \mathrm{f}$
4 Jean Martin/Olivier Guillod (Fn. 8) 2051 /2058.

5 BGE 120 IV 113 E. 2 äussert sich nicht zu der hier diskutierten Frage.

6 Vgl. dazu etwa Martin Schubarth, Therapiefreiheit des Arztes und Selbstbestimmungsrecht des Patienten - Konsequenzen für das Arztstrafrecht und die formula magistralis, AJP 2007, $1089 \mathrm{ff}$.

7 So für das Bankgeheimnis Günter Stratenwerth, BSK-BankG, Basel 2005, Art. 47 N 43 ff. mit Hinweis auf ein Urteil des Bundesgerichtes vom 16.12.1930; für das Geschäftsgeheimnis Martin Schubarth, Kommentar zum schweizerischen Strafrecht, Besonderer Teil, Band 2, Bern 1990, Art. 162 N 17.

8 So setzt sich etwa im Kanton VD die zuständige Aufsichtsbehörde, der Conseil de santé, aus 16 Mitgliedern zusammen, zuzüglich drei Mitarbeiter des Departements mit beratender Stimme bzw. Sekretärfunktion (Loi sur la santé public, LSP, Art. 12). Allerdings werden seine Entscheidungen gemäss Jean Martin/Olivier Guillod, Secret médical/Arztgeheimnis, SÄZ 2000, 2047-54 meistens in Zweierbesetzung getroffen.

9 Art. 12 Abs. 1 lit. c LSP VD.

10 Vgl. dazu Marinette Ummel/Jean-Pierre Restellini, Les instances de levée du secret médical en Suisse, SJZ 1994, 361 ff., 364.

11 Vgl. Alexander Filli, Die Auskunftserteilung des Arztes an Behörden unter dem Aspekt des Berufsgeheimnisses gemäss Art. 321 StGB, BJM 1987, 57 ff., 69; Keller (Fn. 2) 158 ff.

12 Dass und in welchem Ausmass das Arztgeheimnis durch das Krankenkassen- und Versicherungsvertragsrecht faktisch erheblich durchlöchert ist sei im folgenden dahingestellt; vgl. dazu Odile Pelet/Ralph Schlosser, TARMED et le secret médical, in: Mélanges Eric Stoudmann, Zürich 2005, 199 ff.; zur Verletzlichkeit des Arztgeheimnisses auf Grund von Chipkarten, vernetzten Arztpraxen oder in Spitälern Tag 2007 (Fn. 1) 744 und 747.

13 Vgl. dazu das oben (Fn. 7) zitierte Urteil des Bundesgerichtes: Bankgeheimnis hat zurückzutreten, wenn die eigene Existenz der Bank und die Ansprüche ihrer Gläubiger auf dem Spiel stehen. 